\title{
Erratum
}

\section{Erratum: Weible et al., Differential Involvement of Three Brain Regions during Mouse Skill Learning}

In the article "Differential Involvement of Three Brain Regions during Mouse Skill Learning," by Aldis P. Weible, Michael I. Posner, and Christopher M. Niell, which published online on August 1, 2019, an author's name appears incorrectly. Christopher M. Niell should be Cristopher M. Niell. This correction does not affect the conclusions of the article. The name has been corrected in the online record.

https://doi.org/10.1523/ENEURO.0463-19.2019 